\title{
Blockchain-Einsatz zur Optimierung von Produktrückrufen
}

\author{
Tobias Rieke und André Sardoux Klasen, FH Münster
}

\begin{abstract}
Blockchains $(B C)$ werden häufig direkt Bitcoin und andere Kryptowährungen verbunden. Dabei stellt BC die Technologie dar, auf der Bitcoin und Co basieren [1] und sind ein Anwendungsbeispiel unter vielen. Die BC besitzt einige Eigenschaften, die auch für das Supply Chain Management relevant sind. Produktrückrufe nehmen aufgrund der komplexen Supply Chains (SC) immer weiter zu. Dabei liegt die Herausforderung darin, einen Produktrückruf effizient vorzubereiten, durchzuführen und anschließend in die Ursachenanalyse einzusteigen. Genau an dieser Stelle kann die BC-Technologie unterstützen und Transparenz schaffen. So kann eine Reaktion schnell, kosteneffizient und situativ angemessen erfolgen. Ziel des Beitrags ist, einen Einblick in das Potenzial von BC für die Herausforderung „Produktrückruf" zu geben.
\end{abstract}

Eine Blockchain versteht sich als dezentrales System, welches Daten transaktionsorientiert speichert und über seinen Aufbau Manipulationen nahezu unmöglich macht. Kopien der Kerninhalte (das Hauptbuch, engl.: Ledger) liegen verteilt bei jedem (vollständigen) Teilnehmer des Netzwerks. Abgelegte Transaktionen können vielfältig sein und dokumentieren Übertragungen von Eigentum, Finanzströme, Standortdaten, weitere diverse Sensordaten (Temperatur, Feuchtigkeit, Erschütterungen etc.) oder eine Kombination dieser. Die Gültigkeit einer Transaktion wird mithilfe eines Konsensalgorithmus überprüft, mit dem die Wahrscheinlichkeit einer ungültigen Transaktion minimiert wird.

\section{Blockchain: Grundlagen}

Die BC erlaubt eine dezentrale Speicherung von Transaktionen in den verteilten Hauptbüchern, wodurch eine Redundanz der Daten geschaffen wird. Die Daten können durch alle Teilnehmer (je nach Art der BC) im Netzwerk gelesen und geschrieben werden. Die BC stellt im klassischen Sinne keine Datenbank, insbes. keine relationale Datenbank dar. Jedoch ermöglicht die BC über eine Kette von Transaktionen einen Datenbestand aufzubauen und diesen mit jeder weiteren Transaktion zu ergänzen. Bereits erfolgte Transaktionen bleiben dabei erhalten und werden nicht gelöscht oder überschrieben. Ob neue Daten hinzugefügt werden können, wird durch den Konsensalgorithmus sichergestellt, der neue Transaktionen validiert und nicht valide/gültige Transaktionen abweist
[2]. Die Kette der Transaktionen kann in der BC durch die Teilnehmer nachvollzogen werden (Integritätsprüfung). Klassische Datenbanksysteme gewährleisten die Integrität der Daten durch Regeln, die die syntaktische bzw. relationale Korrektheit der Daten sicherstellt. Die Nachvollziehbarkeit der enthaltenden Informationen ist für den Anwender regelmäßig nicht gegeben, da jeweils nur der aktuelle Datenbestand vorhanden ist. Durch Kompromittierung der zentralen Datenbank, können damit bestehende Inhalte verändert werden, ohne dass dieses durch die Nutzer selbst direkt nachvollziehbar ist [3]. In einem Netzwerk sich nicht vollständig vertrauenden Mitgliedern stellt die Nachprüfbarkeit jedoch eine essenzielle Eigenschaft für die Zusammenarbeit dar. Die BC kann eine gemeinsame vertrauensvolle Dokumentationsbasis bereitstellen, die durch jedes Mitglied jederzeit überprüft werden kann. Über Hashwerte der Transaktionen können diese lückenlos nachvollzogen werden [4]. Der Zugang zu dem BC-Netzwerk und der Transaktionshistorie kann allen Teilnehmern (öffentlich) oder nur einem zugelassenen Personenkreis (privat) gewährt werden. Analog kann mit Schreibrechten verfahren werden [5].

\section{Produktrückrufe als Herausforderung von Supply Chains}

Der internationale Handel und der damit verbundene Güterverkehr nehmen weiter zu [6]. Dabei ist die Produktherstellung oft ein Re-

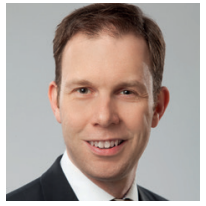

Prof. Dr. Tobias Rieke lehrt und forscht in den Fachgebieten Digitalisierung und Projektmanagement am Institut für Technische Betriebswirtschaft (ITB der FH Münster. Er ist Gründungsmitglied des Instituts für Prozessmanagement und Digitale Transformation (IPD) der FH Münster.

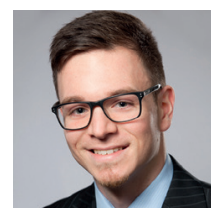

André Sardoux Klasen M. Sc., arbeitet als wissenschaftlicher Mitarbeiter im Fachgebiet Digitalisierung und Mixed Reality am ITB und IPD.

tobias.rieke@fh-muenster.de www.fh-muenster.de/ipd 
[1] Nakamoto, S.: Bitcoin: Ein elektronisches Peer-to-Peer-Bezahlsystem. URL: wWW bitcoin.de/de/bitcoin-whitepaper-deutsch-html, Abrufdatum 15.01.2019.

[2] Chalaemwongwan, N.; Kurutach, W.: State of the art and challenges facing consensus protocols on blockchain. In: The 32 nd ference on Information Networking (ICOIN 2018) (2018), S. 957-962.

[3] Wüst, Karl: Gervais, Arthur: Do you Need a Blockchain? In: 2018 Crypto Valley Conference, S. 45-54.

4] Peck, M. E.: Blockchains: How they Work and Why They'll Change the World. URL: https://spectrum.ieee.org/ computing/networks/blockchains-how-they-work-andwhy-theyll-change-the-worId, Abrufdatum 14.03.2019.

[5] Drescher, D.: Blockchain Grundlagen. Eine Einführung in die elementaren Konzepte in 25 Schritten. 1. Auflage. Frechen 2017.

[6] Arndt, H.: Supply Chain Management. Optimierung logistischer Prozesse. Wiesbaden 2018

[7] Lehmacher, W.: The global supply chain. How technology and circular thinking transform our future. Cham 2017.

[8] Allianz Global Corporate \& Specialty SE: Product Recall. Managing the Impact of the new Risk Landscape. URL: www.agcs.allianz.com/content/dam/onemarketing/ agcs/agcs/reports/AGCS-Product-Recall-Report.pdf, Abrufdatum 15.03.2019.

[9] van Venrooy, M.: Produktrück rufe in der deutschen Automobilindustrie. MotivationsKommunikations- und Verständnisproblematiken. Wiesbaden 2015

[10] Institute of Medicine: Improv ing food safety through a one health approach. Workshop summary. In: National Acad. Press. Washington 2012.

[11] Spiegel Online: Bockshornklee-Samen als Ehec-Verursacher bestätigt. URL: www.spiegel.de/ wissenschaft/mensch/epidemie-bockshornklee-samen-als-ehec-verursacher-bestaetigt-a-80067. html, Abrufdatum 12.08.2018.

[12] Strassmann, B.: Gleitmittel fürs Gaspedal. URL: www.zeit. de/2010/07/T-Toyota, Abrufdatum 15.03.2019.

[13] Focus Online: Toyota-Rückruf wird zum Politikum. URL www.focus.de/auto/news/ klemmendes-gaspedal-toyota-rueckruf-wird-zum-politikum_aid_475335.html, Abrufdatum 15.03.2019.

[14]Casey, M. J.; Wong, Pindar: Global Supply Chains are About to Get Better, Thanks to Blockchain. URL: https:// hbr.org/2017/03/global-supply-chains-are-about-to-getbetter-thanks-to-blockchain Abrufdatum 10.03.2019.

\begin{tabular}{|c|c|c|}
\hline \multicolumn{3}{|c|}{ Beispiele für Rückrufskandale der Vergangenheit } \\
\hline EHEC-Skandal 2011 & Toyota Gaspedal 2010 & E.coli bei Chiptole 2015 \\
\hline $\begin{array}{l}\text { Branche: } \\
\text { Lebensmittel }\end{array}$ & $\begin{array}{l}\text { Branche: } \\
\text { Automobilindustrie }\end{array}$ & $\begin{array}{l}\text { Branche: } \\
\text { Lebensmittel }\end{array}$ \\
\hline $\begin{array}{l}\text { Ursache: } \\
\text { - Mit Keimen kontaminierter } \\
\text { Sprossen in Salaten etc. }\end{array}$ & $\begin{array}{l}\text { Ursache: } \\
\text { - Klemmende Gaspedale }\end{array}$ & $\begin{array}{l}\text { Ursache: } \\
\text { - Mit E.coli Bakterien befallene } \\
\text { Lebensmittel }\end{array}$ \\
\hline $\begin{array}{l}\text { Auswirkung: } \\
\text { - } 53 \text { verstorbene Menschen }\end{array}$ & $\begin{array}{l}\text { Auswirkung: } \\
\text { - } 19 \text { verstorbene Menschen } \\
\text { - Schaden in Milliardenhöhe } \\
\text { - Imageverlust }\end{array}$ & $\begin{array}{l}\text { Auswirkung: } \\
\text { - } 55 \text { erkrankte Menschen } \\
\text { - Einbruch der Aktie um } 42 \%\end{array}$ \\
\hline $\begin{array}{l}\text { Größte Herausforderungen: } \\
\text { - Identifikation der Quelle und } \\
\text { belieferten Kunden } \\
\text { - Identifikation betroffener } \\
\text { Produkte/ Chargen } \\
\text { - Rückruf der entsprechenden } \\
\text { Produkte }\end{array}$ & $\begin{array}{l}\text { Größte Herausforderungen: } \\
\text { - Schnelle Identifikation betroffener } \\
\text { Fahrzeuge } \\
\text { - Global verteilte Werke } \\
\text { - Rückruf der entsprechenden } \\
\text { Produkte }\end{array}$ & $\begin{array}{l}\text { Größte Herausforderungen: } \\
\text { - Identifikation der Quelle } \\
\text { - Zeitnahe Reaktion } \\
\text { - Fehlende Transparenz } \\
\text { - Fehlende Zurechenbarkeit der } \\
\quad \text { Zutaten }\end{array}$ \\
\hline
\end{tabular}

- Es ist wichtig, schnell und effizient die Quelle zu identifizieren.

- Globale Supply Chains sind sehr komplex und mit langsamen, pflegeintensiven Datenbankensystemen im Produktrückruf anfällig.

- Unzureichende Reaktionen können maßgeblich finanziellen Schaden verursachen und Menschenleben riskieren

- Ein geeignetes System, dass Transparenz und Rückverfolgbarkeit in der Supply Chain gewährleistet, ist zwingend erforderlich und hätte Schäden verhindern bzw. verringern können.

Bild 1: Beispiele für Rückrufskandale der Vergangenheit [11-14].

sultat langer und zum Teil zunehmend komplexerer SCs [7]. Eine große Herausforderung stellen hier Produktrückrufe dar, mit teilweise großen finanziellen Auswirkungen. So beziffern sich durchschnittliche Schäden von 2,12 Mio. Euro in der Automobilindustrie, über 1,31 Mio. Euro in der Lebensmittelbranche bis zu durchschnittlich 722.000 Euro in der Verpackungs- und Stahlindustrie [8]. Die Anzahl der jährlichen Produktrückrufe in fast allen Branchen hat sich zudem innerhalb von 15 Jahren (1998-2013) verdreifacht [9].

Besonders die Lebensmittelbranche besitzt häufig äußerst komplexe SCs. Ein Cheeseburger einer Fastfood-Kette besteht aus ungefähr 80 Zutaten, die global produziert und zugeliefert werden [10]. Auch wenn der Lebensmittelbereich von komplexen SCs geprägt ist, finden sich diese auch in weiteren Branchen wieder. Die Herausforderung besteht darin, die Übersicht über die Herkunft der einzelnen Produktkomponenten und dessen Zulieferer zu wahren. Die in Bild 1 dargestellten, in der Presse berichteten "Rückrufskandale“ zeigen die Bedeutung eines adäquaten Systems für Produktrückrufe auf. Dabei werden auch die zentralen Herausforderungen der Produktrückrufe deutlich.

Ein Produktrückruf ist die nachträgliche bzw. proaktive Entfernung von Waren aus dem Markt, um potenzielle Gefahren beim Kunden zu vermeiden [15]. Dafür muss unter anderem ein bidirektionaler Güter-, Wert- und Informationsstrom gewährleistet werden. Bild 2 stellt hierzu einige zentrale Aspekte dar, auf die es bei einem Produktrückruf ankommen kann.

\section{Beitrag der Blockchain für effiziente Produktrückrufe}

Um Ziele des Risikomanagements und der Qualitätssicherung innerhalb einer SC zu erreichen und Produktrückrufe effizient zu managen, bietet sich die BC als Technologie an. Die digitale Nachverfolgung ermöglicht ein Produkt von der Quelle bis zur Senke zu verfolgen [21]. Eine BC erlaubt hierbei, Informationen der SC in Transaktionen zu schützen, um so z.B. eine spätere Manipulation der Dokumentation auszuschließen. Manipulatives Verhalten einzelner Akteure durch die Hinterlegung falscher Angaben in der eigenen Transaktion wird hierbei jedoch nicht verhindert. Zur Zusicherung der korrekten Nachverfolgbarkeit sollten entlang der SC alle Parameter, wie Standort über Zeit, Temperatur und Herkunft einzelner Bestandteile für jedes einzelne, spezifische Produkt zuverlässig, fälschungssicher und transparent dargestellt werden können. Passend dazu wird ein System zur Nutzung von $B C$ und Radiofrequenzidentifikation (RFID) zur Umsetzung einer Gefahrenanalyse und kritischen Kontrollpunkten (engl: Hazard Analysis and Critical Control Points, HACCP) 
in der SC für Nahrungsmittel vorgeschlagen [22]. Die RFID-Transponder dienen der effizienten Identifikation der einzelnen Produkte. Die einzelnen Instanzen der SC können mit Bezug zum RFID-Tag des Produktes verschiedene Parameter als Daten in die BC speichern, z. B. wann sie produziert worden sind, aus welcher Charge sie stammen oder wie die genaue Konfiguration bei sehr variantenreichen Produkten ist. Die nachfolgende Instanz kann aufgrund des Aufbaus einer BC diese Daten nicht mehr ändern und nur eigene Daten ergänzen. Am Ende kann sowohl nachvollzogen werden, an welche Kunden und Standorte die Produkte geliefert worden sind, als auch ihre Herkunft bestimmen [23] und dies zu jeder Zeit an jedem Ort, sofern eine Internetverbindung verfügbar ist. Diese gesicherte Datengrundlage bietet zudem das Potenzial potenzielle Rückrufe nun auch deutlich gezielter durchführen zu können, da die betroffenen Produkte und Kunden deutlich besser identifiziert werden können [24]. Beim Aufbau der BC ist hier der Datenschutz ein zentraler zu berücksichtigender Punkt, sodass vertrauliche Informationen, insbesondere persönliche Kundendaten geschützt sind. Ein beispielhafter Prozessablauf ist in Bild 3 dargestellt.

Ein gutes Beispiel stellt Walmart dar: Walmart testete in Kooperation mit IBM das Potenzial der BC im Rahmen eines Pilotprojekts, basierend auf dem Hyperledger-Framework, für den Schweinefleischmarkt in China. Hintergrund sind erhöhte Sicherheitsbedenken, die bezüglich des Schweinefleischs in China herrschen und die Preise in die Höhe treiben [24]. Ziel war es, die Datensicherheit und -genauigkeit hinsichtlich Herkunft, Chargennummer, Fabrikund Prozessdaten, Ablaufdatum, Lager- und Transporttemperatur und weiterer Transportparameter zu gewährleisten [25]. Durch die Anwendung der Technologie, konnte die Zeit für das Tracking der Herkunft und weiteren Parameter eines einzelnen Produkts von einigen Tagen auf wenige Minuten reduziert und die Reaktionsfähigkeit im Falle eines notwendigen Produktrückrufs enorm gesteigert werden [26]. Aus diesem Grund weitet Walmart das Tracking auf zusätzliche Produkte aus und ruft weitere Zulieferer dazu auf, ebenfalls BC für diesen Zweck einzusetzen [27]. Viele weitere Unternehmen starten Projekte, um die Herkunft der Produkte nachzuweisen [28].

Die oben beschriebenen Erkenntnisse lassen sich auf alle produzierende SCs übertragen [29, 30]. Kunden mit defekten Produkten können so direkt kontaktiert werden. Dabei ist anzunehmen, dass massiv Kosten gespart und Imageverluste zu großen Teilen vermieden werden können [31].

Bild 2: Zentrale Aspekte zum Thema Produktrückruf $[8,16-20]$.

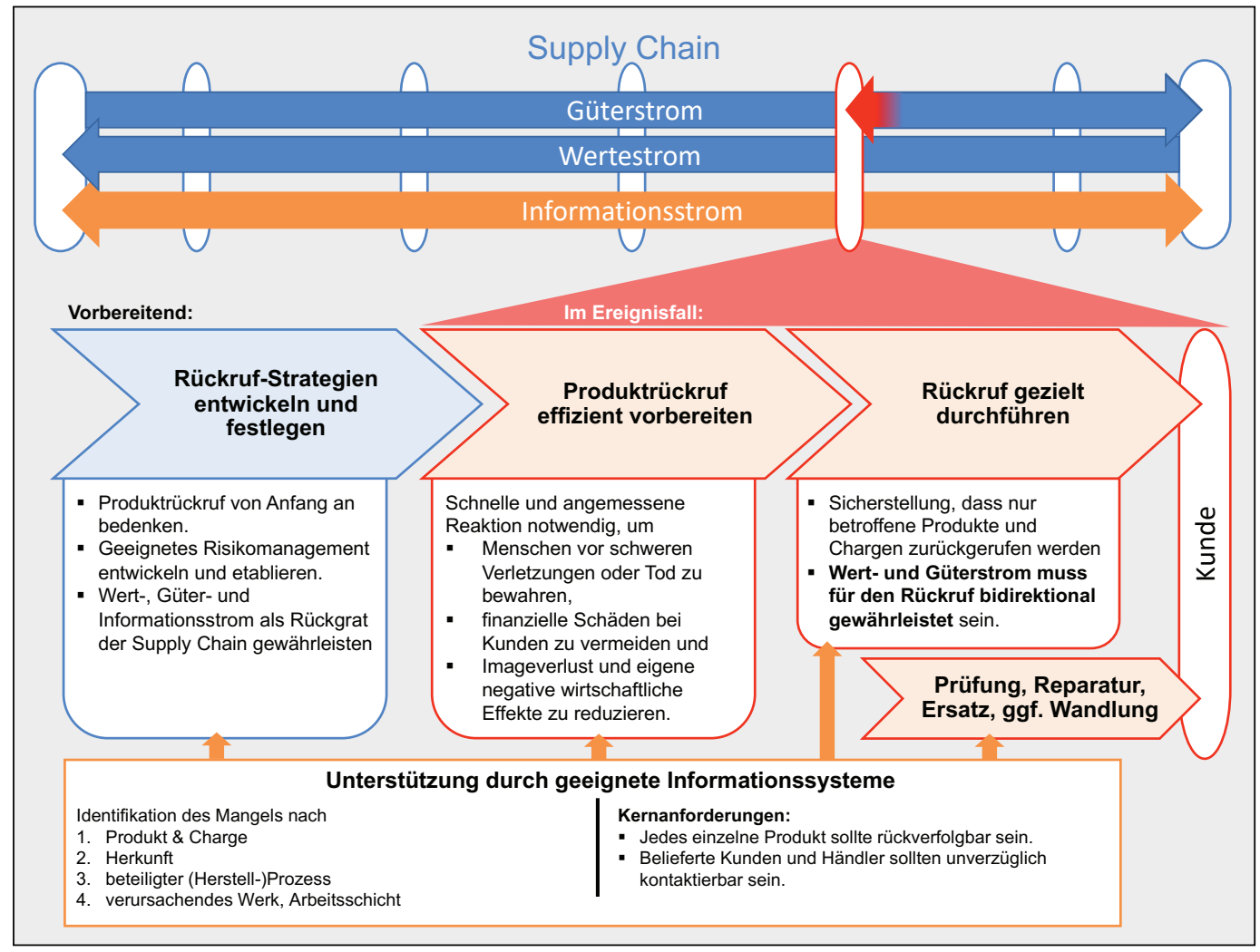

[15] Europäisches Parlament: Richtlinie 2001/95/EG über die allgemeine Produktsicherheit 2001

[16] Zhao, X.; Li, Y.; Flynn, B. B.: The financial impact of product recall announcements in China. In: International Journal of Production Economics 142 (2013), S. 115-123.

[17] Glasl, T: Klindt, T: Krisenfall Produktrückrufe. Erfolgreiches Management - Recht - Kommunikation. Stuttgart 2012.

18] Basole, R. C: Nowak, M: Assimilation of tracking technology in the supply chain. In: Transportation Research Part E: Logistics and Transportation Review 114 (2018), S. 350-370.

[19] Magno, F.: Managing Product Recalls: The Effects of Time, Responsible vs. Opportunistic Recall Management and Blame on Consumers' Attitudes. In: Procedia - Social and Behavioral Sciences 58 (2012), S. 1309-1315.

[20] Craig, S. N.; Thomas, R. J.: A strategic approach to managing product recalls. In: Harvard Business Review 74 (5) (1996), S. 102-112.

[21] Edmund, M.: On the Fast Track? In: Quality Progress 51 (2018) 2, S. 10-12

22] Tian, F.: A supply chain traceability system for food safety based on HACCP, blockchain $\&$ Internet of things. In: The 14th International Conference on Services Systems and Services Management (ICSSSM2017) (2017), S. 16-18.

[23] McDermott, B.: Improving confidence in food safety with IBM Blockchain URL: www.ibm.com/blogs/blockchain/2017/09/improvingconfidence-in-food-safety-with-ibm-blockchain/ Abrufdatum 13.03.2019.

[24] Cai, L.; Pongrace, A.; Butts, Christian; Wang, S.: China's astounding appetite for Pork. Recent trends and implications for international trade. URL https://publicpolicy.wharton.upenn.edu/live/ news/644-chinas-astounding-appetite-for-pork-recent-trends, Abrufdatum 10.03.2019.

[25] del Castillo, M.: Walmart Blockchain Pilot Aims to Make China's Pork Market Safer. URL: www.coindesk.com/walmart-blockchain-pilot-china-pork-market/, Abrufdatum 10.03.2019.

[26] Higgins, S. Walmart: Block chain Food Tracking Test Results Are 'Very Encouraging'. URL: www.coindesk.com walmart-blockchain-food-tracking-test-results-encouraging/, Abrufdatum 13.08.2018.

[27] Walmart: In Wake of Romaine E. coli Scare, Walmart Deploys Blockchain to Track Leafy Greens. URL: https://news. walmart.com/2018/09/24/inwake-of-romaine-e-coli-scare-walmart-deploys-blockchain-to-track-leafy-greens, Abrufdatum 12.03.2019. 
[28] Kshetri, N.; Loukoianova, E. Blockchain Adoption in Supply Chain Networks in Asia. In: IT Prof. 21 (2019) 1, S. 11-15.

[29] Abeyratne, S. A.; Monfared, R. P.: Blockchain Ready Manufacturing Supply Chain using Distributed Ledger. In: IJRET 05 (2016) 9, S. 1-10.

[30] Radocchia, S.: 3 Automotive Consumer Safety Issues Blockchain Will Help Resolve. URL: https://blog chronicled.com/3-automotive-consumer-safety-issues-blockchain-will-help-resolve-2ae 4 b 0 c6ece 5 , Abrufdatum 13.03.2019.

[31] Werner, H.: Supply Chain Management. Grundlagen Strategien, Instrumente und Controlling. 6., aktualisierte und überarbeitete Auflage. Wiesbaden 2017

[32] Weber, Ingo; Xu, Xiwei; Riveret, Régis; Governatori, Guido; Ponomarev, Alexander; Mendling, Jan (2016): Untrusted business process monitoring and execution using blockchain In: International Conference on Business Process Management S. 329-347.

[33] Toyoda, Kentaroh; Mathiopoulos, P. Takis; Sasase, Iwao Ohtsuki, Tomoaki (2017) A Novel Blockchain-Based Product Ownership Management System (POMS) for Anti-Counterfeits in the Post Supply Chain. In: IEEE Access 5, S. 17465-17477.

[34] Kshetri, Nir (2018): 1 Blockchain's roles in meeting key supply chain management objectives. In: International Journal of Information Management 39, S. 80-89.

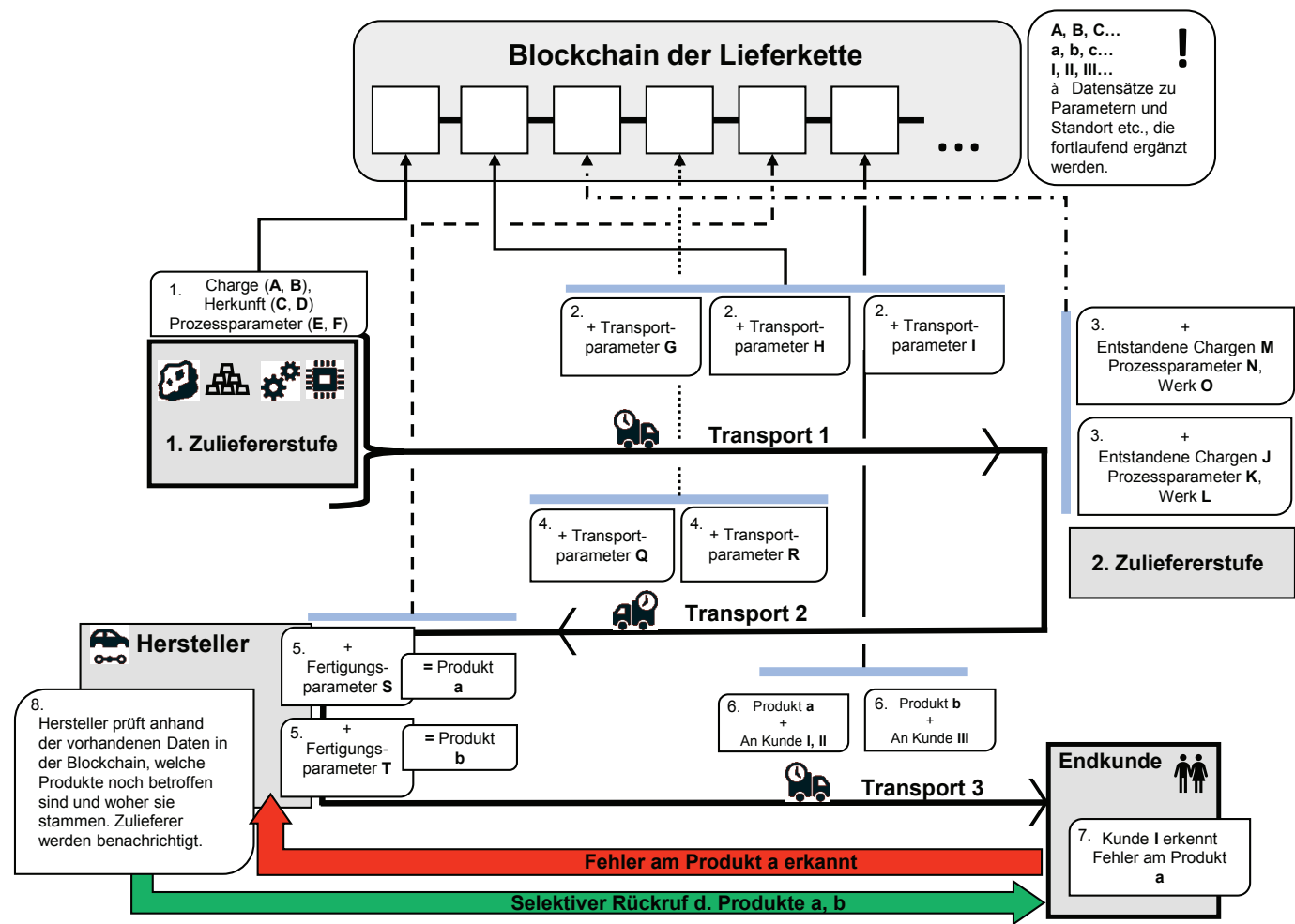

Bild 3: Beispiel einer mit Blockchain unterstützten Supply Chain.

\section{Bewertung des Einsatzpotenzials}

Einige Praxisbeispiele demonstrieren bereits die technische Machbarkeit, eine $B C$ für Produktrückrufe zu verwenden [26-28]. In der Literatur lassen sich konkrete Vorschläge identifizieren, wie eine $\mathrm{BC}$ implementiert werden kann (z.B. [32, 33]). Dennoch bedarf es weiterer Forschung, um auch den ökonomischen Nutzen anhand von realen Einsätzen zu evaluieren. Wenn in der eigenen SC bereits mit RFID-Tags und Sensoren gearbeitet wird, ist der Einsatz einer BC für den Zweck der Nachverfolgung jedoch mit relativ geringen Einstiegskosten verbunden [27]. Dennoch besteht auch an dieser Stelle weiterer Forschungsbedarf, wie eine durchgängige Kette mittels RFID unterstützt werden kann [34] und wie Produkte und Prozesse in der Art gestaltet werden können, dass nicht nur die Dokumentationshistorie fälschungssicherer ist, sondern auch das Produkt. So ist im Einzelfall zu evaluieren, wie z.B. Produkte - möglichst untrennbar - mit RFID-Tags versehen werden können und das Scannen der Tags sowie die Kommunikation mit der BC effizient gestaltet werden kann. Die Herausforderung liegt demnach weiterhin darin, die Schnittstelle zwischen physischer und digitaler Welt fälschungssicher zu machen.
Gleichzeitig stellt die Einführung zudem eine organisatorische Herausforderung dar, da sich der Nutzen erst durch die Beteiligung aller Partner in der SC maximiert. Für die Umsetzung verfügt der Markt über Angebote, um die BC-Technologie zur Verfügung zu stellen (z. B. Hyperledger \& Microsoft Azure). Zudem existieren einige Partner am Markt, die bei der Einführung, insbesondere des für SCs erprobte Hyperledger Fabric, unterstützen (z. B. IBM, SAP, Cisco). Somit kann auf vorhandenem Know-How aufgebaut werden. Im weiteren Vorgehen ist zwingend auf die Art der BC zu achten, die mit verschiedenen Konsensalgorithmen und Zugangsbeschränkungen jeweils Vor- und Nachteile z. B. bzgl. der Skalierbarkeit und Sicherheit des Systems mit sich bringen. Zudem sind die zeitgleich schwierig zu erreichenden Eigenschaften der Transparenz (der Nachverfolgbarkeit) und Anonymität wichtig für die Wahl der BC. Auch wenn das Potenzial der $B C$ für Produktrückrufe groß ist, bleibt eine Einzelbetrachtung und Evaluation zwingend erforderlich.

\section{Schlüsselwörter:}

Blockchain, Supply Chain Management, Produktrückrufe, Lieferkette 